\title{
MODIFICATION OF A CHARGED-BOSE-GAS MODEL FOR OBSERVED ROOM-TEMPERATURE SUPERCONDUCTIVITY IN NARROW CHANNELS THROUGH FILMS OF OXIDISED ATACTIC POLYPROPYLENE
}

\author{
D.M. EAGLES \\ 19 Holt Road, Harold Hill, Romford, Essex RM3 8PN, England \\ e-mail:d.eagles@ic.ac.uk
}

[International Journal of Modern Physics B 25, 1845-1875 (2011)]

\begin{abstract}
Reasons have been found for thinking that the minimum diameter of channels of a given length to support superconductivity at room temperature through films of oxidised atactic polypropylene (OAPP) is considerably larger than found in a model for Bose condensation in an array of nanofilaments [D.M. Eagles, Phil. Mag. 85, 1931 (2005)] used previously. This model was introduced to interpret experimental results dating from 1988 on OAPP. The channels are thought to be of larger diameter than believed before because, for an N-S-N system where the superconductor consists of an array of single-walled carbon nanotubes, the resistance, for good contacts, is $R_{Q} / 2 N$, where $N$ is the number of nanotubes and $R_{Q}=12.9 \mathrm{k} \Omega$ [See e.g. M. Ferrier et al., Solid State Commun. 131, 615 (2004)]. We assume this would be $2 R_{Q} / N$ for a triplet superconductor with all spins in the same direction and no orbital degeneracy, which may be the case for nanofilaments in OAPP. Hence one may infer a minimum number of filaments for a given resistance. In the present model, the $E(K)$ curve for the bosons is taken to be of a Bogoliubov form, but with a less steep initial linear term in the dispersion at $T_{c}$ than occurs at low $T$. This form is different from the simple linear plus quadratic dispersion, with a steeper initial slope, used in my 2005 paper. A combination of theory and experimental data has been used to find approximate constraints on parameters appearing in the theory.

Keywords: one-dimensional sytems - high-temperature superconductivity - oxidised atactic polypropylene -Bose gas - bipolarons - nanofilament arrays
\end{abstract}

\section{Introduction}

There were claims in a 47-page deposited paper in Russian in 1988 that superconductivity occurs at room temperature in narrow channels through thin films of oxidized atactic polypropylene (OAPP) 11, and claims of this in physics journals from 1989 2. The first indications of possible superconductivity at room temperature came from experiments involving the resistance between pressure microcontacts on the top of OAPP films and a conducting substrate. Three 
types of contact points were found, insulating points, points with medium resistance, and points with low resistance. (See Fig. 1, which is based on Fig. 17 of Ref.[1], and has also been published in Ref.[3]). The resistances of the group of low-resistance points lay in the same range for two widely different film thicknesses, making it plausible that the resistance was entirely contact resistance, with negligible resistance in the channels between the contacts and the substrate at these points.

More convincing evidence of room-temperature superconductivity came in 1990 and 1991 from experiments which showed (i) that the conductivity of the low-resistance channels was several orders of magnitude larger than that of $\mathrm{Cu}$ [4, 5], (ii) that the high conductivity was destroyed in pulsed measurements by non-thermal means when currents above a critical value were applied [5], and (iii) there was a negligible electronic contribution to the thermal conductivity, implying a violation of the Wiedemann-Franz law by several orders of magnitude [6]. There are some questions in the present author's mind about how the results of the four-probe measurements of conductivity reported in Ref. [4] came about, but the estimate of the conductivity by indirect methods in Ref. [5] seems soundly based. This estimate of conductivity made use of the thermal conductivity results of Ref. [6], and depended on the lack of decomposition of the sample after repeated pulsed measurements, which permitted a deduction of conductivity greater than $10^{11} \mathrm{ohm}^{-1} \mathrm{~cm}^{-1}$, and a transition temperature from the highly conducting state under pulsed-current conditions of at least $700 \mathrm{~K}$, the decomposition temperature of the polymer. Similar very high superconducting $T_{c}$ 's have been inferred by Zhao and coworkers 17, 8, 9, 10, from conductivity and other measurements on carbon nanotubes, following a suggestion by Tsebro et al. [11] that there might be superconductivity at room temperature in carbon nanotubes. Work claiming possible room-temperature superconductivity in highly oriented pyrolytic graphite 12,13 may be related. Another claim of room-temperature superconductivity in a quasi one-dimensional system has been made by Djurek et al. 14, 15, 16. In Djurek's work, the main material involved, $\mathrm{Ag}_{5} \mathrm{P}_{2} \mathrm{O}_{6}$, contains quasi one-dimensional channels, but in recent work 15, 16] it is found that indications of room-temperature superconductivity occur only when pellets of $\mathrm{Ag}_{5} \mathrm{P}_{2} \mathrm{O}_{6}$ are embedded in an insulating matrix.

Unusual magnetic properties have also been reported in OAPP and other polymers. These are: 1. Metamagnetic transitions at magnetic fields of the order of 1000-4000 Oersted [17, 2, 18, 19, 20] in films of both OAPP and polydimethylsiloxane (PDMS); 2. Large diamagnetism in some films of OAPP 1, 2, 20]; 3. Occasional spontaneous sudden jumps of films of OAPP towards low-field regions in inhomogeneous magnetic fields [18. The metamagnetism is thought to be due either to a transition from an antiferromagnetic to a ferromagnetic arrangement 1, 17, 2] or to alignment of ferromagnetic domains of ferromagnetic (and superconducting) filaments. We take the second viewpoint in this paper. The large diamagnetism and spontaneous jumps to low-field regions in inhomogeneous magnetic fields are thought to be associated with superconducting channels which join at their ends to form closed loops [1, 2, 18, 20, 21]. In Ref. [21] the large diamagnetism observed in two samples of OAPP 20] has been in- 


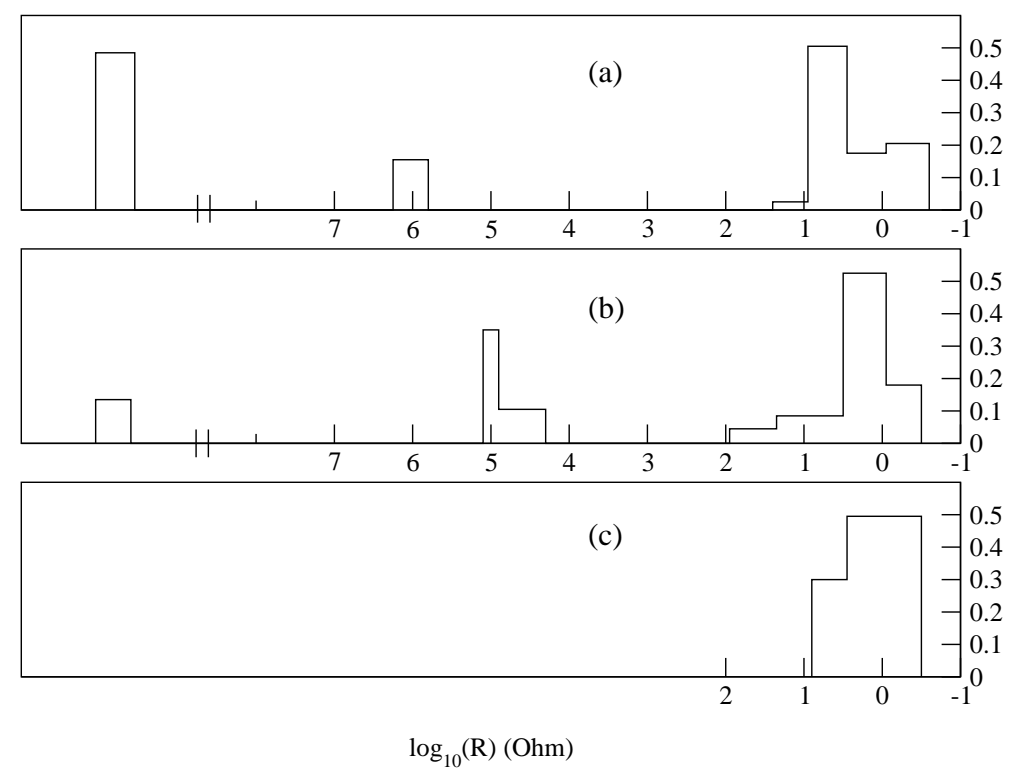

Figure 1: Distribution function for resistance $R$ at room temperature from microprobes to a metallic substrate: (a) For a sample of oxidised atactic polypropylene (OAPP) of thickness $50 \mu \mathrm{m}$, (b) for OAPP with thickness $0.3 \mu \mathrm{m}$, and (c) for the metallic substrate without any polymer film. [Reproduced from Fig. 1 of Ref.[3], which was obtained approximately from Fig. 17 of Ref.[1] 
terpreted in detail as due to induced currents in closed loops, using a model with loops of two different radii as an approximation to a continuous distribution.

Almost no attempts to repeat and extend the results mentioned above have been reported in the literature. At one point, some members of the Ioffe Institute group who reported results supporting superconductivity at room temperature in 1990, decided that the effects observed were due to formation of a conducting filament of the electrode material in high electric fields 22, 23, but such an interpretation has been shown to be untenable for the samples studied by the group of Grigorov[18, 19]. Also, one of the Ioffe Institute group in more recent papers appears to have realised that high electric fields are not needed to produce highly conducting channels through polymer films 24, 25, 26]. Another member of the original Ioffe group, now in Israel, has found switching to high-conductivity states in channels through films of PDMS [27.

Baran et al. found no evidence of unusual magnetic effects in bulk atactic polypropylene 28, but in this case the oxidation was probably not great enough, except perhaps near the surface, for conducting channels to form. I have been informed that a verbal report on some of the samples of Grigorov et al. was given in the 1990's to M. Goldes (CEO of the company with which Prof. Grigorov was associated at the time) by P.M. Grant, who concluded that there was no superconductivity. However, since no details are available, the reliability of this assessment cannot be judged. Dr. Grant wrote a spoof essay about the disovery of superconductivity at $T>541 \mathrm{~K}$ in polymers in 2028 in Physics Today 29, but in my view put the date at about 40 years after the real event! Professor Grigorov appears to have stopped publication on the subject since 2000, concentrating instead on improving samples etc., and patent work [30, 31, 32, and so, unless another group takes up studies in the field, it may be a long time before his work is properly assessed.

Only one group, originally at the Institute of Synthetic Polymeric Materials in Moscow, has consistently claimed room-temperature superconductivity in channels through films of OAPP, PDMS and other polymers, including polyoctylmethacrilate 33. However, an early paper from the Ioffe Institute, and also more recent work by some scientists from the Ioffe Institute, especially Ionov, who was a member of the original group publishing in 1990, collaborating with others from Russia and Germany, have found evidence that superconductivity occurs in narrow channels through many polymer films at temperatures below the $T_{c}$ of superconducting electrodes, and that this is not due to the superconducting proximity effect. Such evidence includes conductivity greater than $10^{14} \Omega^{-1} \mathrm{~cm}^{-1}$ in channels through films between superconducting Sn electrodes 4, Josephson-like I-V curves for superconductor-polymersuperconductor sandwiches in OAPP 4, PDMS 24], poly (phthalidylidene biphenyline) [34, polyamidine 25, and two other polymers [35], oscillations of resistance as a function of magnetic field in films of polyimide, poly (phthalidylidene biphenylene) and PDMS below the $T_{c}$ of Sn 36, 24, 34, and a critical current for a destruction of Josephson-like $I-V$ curves which depends on temperature in a way inconsistent with superconductivity due to the proximity effect in PDMS [24] and poly(phthalidylidene biphenylene) [34, 37]. There is also a 
large number of papers by the group of A.N. Lachinov in Ufa on highly conducting channels through polymer films (see e.g. [38, 39] or a review [40]), but, except in recent work with Ionov and others 41, without suggestions of superconductivity. The work from 1998 onwards sometimes made use of spin-coated films, which method of production allows better control of film thickness 41]. Recent work of Ionov and Rentzsch [42, 43] considers the possibility of resonant tunnelling to explain their results. A difference between results of Ionov et al and those of Grigorov's group is that Ionov and coworkers report a dependence of conductance of channels on the work function of the substrate 44, whereas no such dependence is reported by Grigorov et al.. Possibly the difference is related to how good the contacts with the substrate are. A detailed discussion of different forms of $I-V$ curves for different types of contacts to OAPP and PDMS films is given by Kraev et al. 45.

A theory for why conducting channels exist in films of elastomers (materials with very low elastic constants) has been developed by Grigorov and coworkers 46, 47, 48. The theory involves the formation of one-dimensional channels with diameters of the order of nanometers due to the formation of "superpolarons", i.e. one-dimensional strings of a certain type of polaron involving electrons interacting with rotatable dipolar groups. An unusual theory for superconductivity in such channels involving motion of oppositely directed charge density waves was developed by Grigorov [49, 50. An earlier theory by me for the superconductivity involving large enhancements of pairing interactions at certain high drift velocities 51, 52 has had to be abandoned, because recent work on bipolarons in one dimension 53 gives no support for the greatly enhanced interactions at such velocities predicted by perturbation-type methods. However, work 3 involving Bose condensation of bipolarons (without any special enhancement of interactions at high drift velocities), with an initial linear dispersion at low wave vectors may be on the right track, and the purpose of this paper is to improve on the model introduced in 2005, making more use of both experiment and theory to help to determine parameters in the theory.

The model and constraints we use differ in four ways from that of 2005. First the minimum number of nanofilaments required to support superconductivity at room temperature is taken to be considerably higher than in the 2005 paper. This is because we have realised that, in the case of superconducting channels consisting of single-walled nanotubes between normal-metal electrodes, there is a minimum resistance equal to $R_{Q} / 2 N$ for singlet superconductivity, where $N$ is the number of nanotubes [54, and $R_{Q}=12.9 \mathrm{k} \Omega$ is the resistance quantum. For nanotubes there is an orbital degeneracy as well as the spin degeneracy, which gives rise to the factor of two in the denominator of the above expression. For nanofilaments with no orbital degeneracy and triplet superconductivity with all spins in the same direction, as is thought to be the case for OAPP, we assume that $R_{Q} / 2 N$ becomes $2 R_{Q} / N$, where $N$ is the number of nanofilaments.

The second difference from 2005 arises since we doubt if the form of dispersion for bosons assumed in 2005 based on a Cooper-pair model [55] is appropriate in the Bose-gas regime, where the Fermi surface no longer exists even above $T_{c}$. We assume instead that the dispersion has a Bogoliubov-type form, but with the 
strength of the initial linear slope at $T_{c}$ (related to an effective bosonic chemical potential) smaller than at low $T$. This hypothesis is made as an attempt to account for the fractional increase in $T_{c}$ of an interacting Bose gas due to interactions, proportional to $n_{b}^{1 / 3}$ for low boson concentrations $n_{b}$ [56, 57, in a model of non-interacting bosons with a different type of dispersion from the bare one, in a similar spirit to Fermi-liquid theory. At higher boson concentrations the fractional change of $T_{c}$ of an interacting Bose gas due to interactions decreases with $n_{b}[58$. This can be taken into account in a non-interacting boson model by a mass which increases with $n_{b}$, but with a dependence on $n_{b}$ with a higher power than $1 / 3$. Although there is no a priori reason to choose a Bogoliubov-type of dispersion at $T_{c}$, a possible justification is that above $T_{c}$ there are fluctuating regions of superfluid which support this type of dispersion locally, in analogy to the Aslamozov-Larkin theory [59] for BCS superconductors. The steepness of the initial linear term, proportional to the square root of the effective bosonic chemical potential, is chosen to give approximate agreement of $T_{c}$ for the non-interacting bosons with a Bogoliulobov-type of dispersion with numerical calculations mentioned above for an interacting Bose gas at low boson concentrations. Although the Bogoliubov dispersion is modified in a charged Bose gas (see e.g. 60]), we do not expect great changes from the simple Bogoliubov form at temperatures close to the condensation temperature because screening will cause the interactions to become short range at boson concentrations of interest.

The third difference from 2005 is that we make more use of theoretical work (see especially [4] ) to put constraints on the many parameters in the theory. We determine parameters by minimising a weighted sum of moduli of differences of two sides of equations given by constraints, with the weighting varied according to how accurate we think the constraints are - several of these are thought to be at best only of order-of-magnitude accuracy. We estimate the linear carrier concentration for the $50 \mu \mathrm{m}$ film from the theory of Grigorov 48 which minimises the energy of a ferromagnetic nanofilament, but, in the $0.3 \mu \mathrm{m}$ film we use the linear carrier concentration as an adjustable parameter because charge injection from the electrodes may influence the carrier concentration for films of thicknesses less than about $3 \mu \mathrm{m}[61$. (see also Ref. 62, although the thickness to which this may occur does not appear to be discussed in this reference), and this injection may either increase or decrease the total electron concentration.

There are twelve parameters which need to be determined in our theory. These are: 1) and 2) The numbers $n_{T}$ of nanofilaments in each transverse direction of assumed square arrays of nanofilaments for the highest-resistance superconducting channels for 50 and $0.3 \mu \mathrm{m}$ films; 3) The lattice constant $a_{T}$ of these arrays; 4 ) and 5) The product $c_{B} a_{T}$ of the linear boson concentration $c_{B}$ in a nanofilament with this lattice constant in $50 \mu \mathrm{m}$ films, and the change $\Delta c_{B} a_{T}$ of this product in the $0.3 \mu \mathrm{m}$ films because of possible effects of carrier injection from electrodes; 6 ) The ratio of $a_{T} / \rho$ of $a_{T}$ to the radius $\rho$ of the cylinder around a nanofilament where all dipolar groups are pointing away from the axis of the nanofilament; 7) and 8) The binding energy $E_{p b}$ and the longitudunal mass $M_{L}$ 
of the pairs which form in the polaron string due to mediation of high-frequency excitations; 9) The ratio $p=M_{L} / M_{T}$ of the longitudinal and transverse masses $M_{L}$ and $M_{T}$ of bosons in a nanofilament array; 10) The energy of the excitation which mediates the pairing attraction between the polarons within the superpolaron array, which excitations we assume for most of the paper to be plasmons; 11) The effective bosonic chemical potential $\mu_{B}$ which appears in the assumed Bogoliubov form of the boson dispersion at $T_{c}$; and 12) The reduction $\Delta T_{1}$ of the superconducting transition temperature for the highest-resistance superconducting channel for the $50 \mu \mathrm{m}$ films due to phase slips. We do not have a method of determining the last parameter without a detailed theory of phase-slip resistance for wires with BEC-type superconductivity, but, assuming $T_{c}$ remains above room temperature for all cross sections larger than the minimum required to support superconductivity at that temperature, we can determine a lower limit on $\Delta T_{1} \approx 7 \mathrm{~K}$ within the framework of our model. We do most of our calculations with $\Delta T_{1}=15 \mathrm{~K}$, not far above the lower limit of about $7 \mathrm{~K}$.

After writing down the basic equations of our model in section 2, in section 3 we find values for the first two parameters from Fig. 1 assuming good contacts. Then, from a combination of theory and use of experimental data, sections 2 and 4 to 11 enable us to find nine equations of varying degrees of approximation to be satisfied by parameters 3 ), 4), and 6) to 11 ), assuming a value of $15 \mathrm{~K}$ for $\Delta T_{1}$. In section 12 we find the change of $c_{B} a_{T}$ for the thinner films to make $T_{c}$ equal to room temperature for the $n_{T}$ for the highest-resistance superconducting channel for these films.

Some of the equations are thought to be fairly accurate while others are at best order-of-magnitude constraints. For our numerical work to determine parameters in section 13 we weight the various constraints according to our guesses at their accuracy. Some discussion is given in section 14 .

\section{Model of Non-interacting Bosons in an Array of Nanofilaments with a Bogoliubov Type of Dispersion at $T_{c}$}

As in Ref. [3], we use a model of conducting channels composed of noninteracting bosons on a square lattice forming a square array of quasi onedimensional filaments of length $L$ and transverse dimension $D$ measured from a point half a filament separation $a_{T}$ beyond the centers of the edge filament rows. The filaments are assumed to be sufficiently narrow that the bosons in a filament are in the lowest possible state with respect to motion perpendicular to the filaments at all temperatures of interest. We suppose that boson wave functions vanish at the edges of the array, and neglect any periodicity in the potential along the length of the array. Then we suppose that the boson states in the array can be characterised by the magnitudes of wave numbers $K_{1}, K_{2}$ and $K_{3}$ in the two transverse and longitudinal directions, where $K_{1}$ and $K_{2}$ take on discrete values ranging from $\pi / D$ to $n_{T}(\pi / D)$, and $K_{3}$ takes on values of $k \pi / L$, where $k$ runs from 1 to $\infty$. For computational purposes we approximate the sum to infinity by a finite sum with a suitably high upper limit. 
The difference in our model from that used in Ref. [3] is in the form of the $E_{\mathbf{K}}$ chosen for the bosons. Here we assume that the boson energy $E_{B}\left(K_{1}, K_{2}, K_{3}\right)$, where $K_{1}=i \pi / D, K_{2}=j \pi / D, K_{3}=k \pi / L$, has the Bogoliubov form

$$
\begin{aligned}
& E_{B}\left(K_{1}, K_{2}, K_{3}\right)= \\
& \quad\left[\begin{array}{ll}
\left\{A_{L}(\pi / L)^{2}\right. & \left.\left.\left[\left(k^{2}-1\right)+p(L / D)^{2}\left(i^{2}+j^{2}-2\right)\right]+\mu_{B}\right\}^{2}-\mu_{B}^{2}\right]^{\frac{1}{2}} .
\end{array}\right.
\end{aligned}
$$

Here

$$
A_{L}=\hbar^{2} / 2 M_{L},
$$

and

$$
p=M_{L} / M_{T},
$$

where $M_{L}$ and $M_{T}$ are the boson masses in the longitudinal and transverse directions; $\mu_{B}$ is an effective chemical potential of the bosons at the temperature being considered. We say an effective bosonic chemical potential because the actual bosonic chemical potential at the Bose-condensation temperature must be zero, but, as mentioned in the Introduction, we suppose that interaction between bosons may be taken into account by a Bogoliubov form of dispersion for non-interacting bosons which give about the same type of increase in $T_{c}$ as accurate theories of the effect of interactions find. The same type of model with a simple quadratic dispersion was discussed in Ref. [63, but with the difference from the case assumed here that a discrete lattice was considered in the longitudinal as well as transverse directions.

Condensation into the lowest-energy state cannot take place if the sum of the boson occupation factors over all states except the lowest for a chemical potential situated at the energy of the lowest state is greater than the total number of bosons, $N_{B}$. Below the temperature at which this sum is equal to $N_{B}$, the occupation of the ground state and of states in its neighbourhood will start to become macroscopic, i.e. of the order of $N_{B}$, and so the equality of the sum with $N_{B}$ determines the condensation temperature $T_{B}$. For an average linear concentration $c_{B}$ of bosons per filament, this criterion can be written as

$$
\sum_{K_{1}, K_{2}, K_{3}} \frac{1}{\exp \left[E_{B}\left(K_{1}, K_{2}, K_{3}\right) / k_{B} T_{B}\right]-1}=c_{B} L n_{T}^{2},
$$

where the sums over $K_{1}$ and $K_{2}$ are sums from $i, j=1$ to $n_{T}$ of $K_{1}=i \pi / D$ and $K_{2}=j \pi / D$, and the sum over $K_{3}$ is a sum from $k=1$ to infinity of $K_{3}=k \pi / L$, but the ground level $i=j=k=1$ is excluded.

As discussed in Ref. [3], it is difficult to define unambigously a condensation temperature for small arrays because of the gradual way in which occupation numbers of the ground state change with temperature for such arrays [64, 65]. However, we shall be concerned here mainly with arrays containing more than about 10,000 bosons, and so this ambiguity should not be a problem.

\section{Constraints from Histograms of Resistances with Microcontacts}

Because of the lack of dependence of the average resistance on film thickness 
of the lowest-resistance parts of the histograms in Fig. 1, and for other reasons discussed in the Introduction, we assume that the channels associated with these parts of the histograms are superconducting at room temperature. Further, based on previous studies 63 , 3] which found that, at least for fairly low cross sections of channels, the Bose condensation temperature of nanofilament arrays increases with the array cross section, we suppose that the superconducting $T_{c}$ tends to increase as the resistance measured by the two-point probe method decreases. Hence we assume that the highest-resistance channels of the superconducting group (HRSC's) have superconducting $T_{c}$ 's of just about room temperature, i.e. we assume that

$$
T_{c, H R S C}(L) \approx 295 K,
$$

where $L=50 \mu \mathrm{m}$ or $0.3 \mu \mathrm{m}$ for the highest-resistance channels of the lowresistance groups in Figs 1(a) and (b) respectively.

For an array of single-walled superconducting carbon nanotubes with good contacts from normal metals, there is a minimum resistance 54 .

$$
R_{m}=R_{Q} / 2 N,
$$

where

$$
R_{Q}=12.9 \mathrm{k} \Omega
$$

is a resistance quantum. Single-walled carbon nanotubes have two orbitallydegenerate and spin-degenerate subbands [54, 66. We assume that this minimum resistance becomes $2 R_{Q} / N$ for triplet superconductivity in nanofilaments with all the spins of a nanofilament in the same direction, as is thought to be the case for OAPP [46, 47, 48]. We have no reason to assume orbitallydegenerate subbands, and, if all spins are aligned, this also halves the number of current paths. Hence, assuming good contacts, we suppose that $n_{T}$ for the highest-resistance superconducting channels for the two film thicknesses being considered satisfies

$$
n_{T, H R S C}(L)^{2} \approx 2 R_{Q} / R,
$$

where $R$ is the resistance for the relevant channel. Using data from Fig. 1 we deduce

$$
n_{T, H R S C}(0.3 \mu \mathrm{m}) \approx 17, n_{T, H R S C}(50 \mu \mathrm{m}) \approx 32 .
$$

For samples with small cross sections, phase slips cause resistance 67, 68]. Defining, arbitrarily, $T_{c}$ of the system as that temperature at which phase slips cause a resistance of half the resistance in the normal state, then phase slips reduce an initial superconducting transition temperature $T_{c 0}$ to $T_{c}$, with

$$
T_{c}=T_{c 0}-\Delta T
$$

where, for BCS-type superconductors 67,

$$
\Delta T \propto(\text { cross }- \text { section })^{-2 / 3} \propto \mathrm{n}_{\mathrm{T}}^{-4 / 3} .
$$


Although there are papers on phase slips for Bose condensates [69, 170, they do not appear to give sufficient information to determine the dependence of $\Delta T$ on $n_{T}$ or on coherence lengths for such systems. Thus we use Eq. (11) to find the dependence of $\Delta T$ on $n_{T}$ for a given boson concentration in our numerical work.

\section{Relation between Linear Boson Concentration and Other Param- eters based on Grigorov's 1991 theory}

In an elastomer (a material with very low elastic moduli) containing rotatable groups of atoms with electric dipole moments, an excess electron causes all dipoles to point away from the electron within a radius $R_{0}$ given by 46 ]

$$
R_{0}=\left(e D_{m} / \pi v G \epsilon_{h}\right)^{\frac{1}{2}}
$$

forming an unusual type of polaron. Here $D_{m}$ is the dipole moment of a group, $v$ is its volume, $G$ is a shear modulus, and $\epsilon_{h}$ is a high-frequency dielectric constant. For many electrons in such a material it is energetically favourable for the polarons to form strings called "superpolarons" 46, 47, 48. For superpolarons with a linear concentration of polarons $c_{F}$, the dipoles point outward in a cylinder of radius $\rho=2 R_{0}^{2} c_{F}$ [4]. In our theory we suppose that there is pairing of polarons within a string caused by attraction mediated by plasmons or high-energy phonons, to give a linear concentration of bosons $c_{B}=0.5 c_{F}$, and so, putting results in terms of boson concentrations we have

$$
\rho=4 R_{0}^{2} c_{B}
$$

When exchange forces are included, Grigorov 48, finds that, for low carrier concentrations, the minimum energy of a polaron string occurs when the polarons are ferromagnetically aligned with a linear concentration such that

$$
k_{F} R_{0} \approx 1.6
$$

where $k_{F}$, the Fermi wave number for spin-aligned electrons, is

$$
k_{F}=\pi c_{F}=2 \pi c_{B} .
$$

Ferromagnetism at low concentrations in a quasi one-dimensional electron gas has also been discussed by Shelykh et al. [71.

We consider a square array of polaron strings (to be referred to as nanofilaments from now on) with lattice constant $a_{T}$. From Eqs. (13-15), we find that

$$
c_{B} a_{T}=4 \times(1.6 / 2 \pi)^{2}\left(a_{T} / \rho\right)=0.259\left(a_{T} / \rho\right) .
$$

5. Relation between Separation of Nanofilaments and Radius of Fully Aligned Dipole Region from Experimentally Based Estimates of Average Carrier Concentration in Conducting Channels 
The average concentration of bosons within channels is not known accurately. However, we may obtain an order-of magnitude estimate of this as follows. From magnetisation results, the maximum concentration of aligned spins in any sample reported on up till now appears to be that for sample 1 of Ref. 20, where the saturation magnetisation after any diamagnetic contribution has been removed by field cycling is $0.058 \mathrm{emu} \mathrm{cm}{ }^{-3}$ (see dotted curve in Fig. 1 of Ref. 20]). Hence, assuming this moment is due to aligned electron spins, the average concentraion $n_{F}$ of fermions in this sample is given by

$$
n_{F} \approx 6.3 \times 10^{18} \mathrm{~cm}^{-3} .
$$

The highest concentration of conducting channels reported in any sample is given in Ref. [72, where the average separation of the channels was determined to be $7.5 \mu \mathrm{m}$. Hence with a typical channel diameter of about $[5] 1 \mu \mathrm{m}$, the ratio $V_{s} / V_{c}$ of the volume of the sample to the total volume occupied by conducting channels is given by

$$
V_{s} / V_{c} \sim 7.5^{2} \times(4 / \pi)=72 .
$$

If we suppose that the fraction of material occupied by channels in sample 1 of Ref. [20] is about the same as in the sample of Ref. [72, then, from Eqs. (17) and (18), we deduce that the concentration of bosons $n_{B}$ in the channels (half the fermion concentration) satisfies

$$
n_{B} \sim 2.2 \times 10^{20} \mathrm{~cm}^{-3} .
$$

Using

$$
n_{B}=\left(c_{B} a_{T}\right) a_{T}^{-3}
$$

and Eqs. (16) and (19), we deduce that

$$
a_{T} \approx 1.04\left(a_{T} / \rho\right)^{1 / 3} \mathrm{~nm} .
$$

Although we used $\sim$ in Eq. (19), we have changed to $\approx$ in Eq. (21) because of the one-third power.

\section{Estimate of Transverse Bipolaron Mass based on Grigorov's 1991 Theory}

In this section we make use of the theory of Ref. 48 to estimate the transverse mass for motion of particles across our array before inclusion of pairing due to mediation of plasmons or high-frequency phonons, and use these calculations to estimate the ratio $M_{L} / M_{T}$ of longitudinal and transverse bipolaron masses in terms of other parameters. Assuming a tight-binding model for transverse motion across the array, the transverse single-particle masses can be found in terms of energy overlap integrals $t$.

We suppose that the diameter $D$ of the neutral cylinder enclosing a polaron string in the theory of Ref. [48] can be identified with $a_{T}$ for our array of strings. The potential energy appearing in an expression for $t$ has to be measured from 
a zero equal to the lowest energy state of an isolated string. This energy, which we call $E_{0}$, is given by

$$
E_{0}=2 \pi \int_{0}^{\infty}\left[\chi^{2}(r) V(r)-\left(\hbar^{2} / 2 m_{e}\right) \chi d^{2} \chi / d r^{2}\right] d r,
$$

where the (real) radial wave function $\chi(r)$ is given by

$$
\chi(r)=(1 / 2 \pi)^{1 / 2}(2 / 3) 2^{1 / 2} \beta(1+\beta r) \exp (-\beta \mathrm{r}),
$$

with

$$
\beta=5 / R_{0}=5 \times 2 \pi c_{B} / 1.6=19.6 c_{B}
$$

in our notation. We have changed slightly from Grigorov's notation in that we have included a factor $(1 / 2 \pi)^{1 / 2}$ in $\chi$. The potential energy of an electron $V(r)$ in our notation satisfies

$$
\begin{array}{r}
V(r)=-\left[e^{2}(1-k) /\left(\epsilon_{h} a_{T}\right)\right] \\
\left\{2 c_{B} a_{T}\left[2 \ln \left(a_{T} / 2 \rho\right)+\left(2 \rho / a_{T}\right)^{2}-1\right]+a_{T}(\rho-r) / R_{0}^{2}\right\} \quad(r<\rho) \\
=-\left[2 e^{2}(1-k) c_{B} a_{T} /\left(\epsilon_{h} a_{T}\right)\right]\left[2 \ln \left(a_{T} / 2 r\right)+\left(2 r / a_{T}\right)^{2}-1\right] \quad\left(\rho<r<a_{T} / 2\right) \\
=0 \quad\left(a_{T} / 2<r\right) .
\end{array}
$$

Here

$$
k=\epsilon_{h} / \epsilon_{s},
$$

where $\epsilon_{s}$ is the static dielectric constant, and $\epsilon_{h}$, is the high-frequency dielectric constant. For materials in which polaron strings occur, $k$ is small compared with unity.

For wave functions as given by Eq. (23), we find that the energy overlap integral $t$ is given by

$$
\left.t=\int_{0}^{\infty} \int_{0}^{2 \pi} 2 \pi r\left[V(r)-E_{0}\right)\right] \chi(r) \chi(u) d r d \theta,
$$

with

$$
u=\left[a_{T}^{2}+r^{2}-2 a_{T} r \cos \theta\right]^{1 / 2} .
$$

The bare transverse mass $m_{F T b}$ of fermions for our square lattice in a tightbinding model is related to the energy overlap integral $t$ by

$$
m_{F T b}=\hbar^{2} / 2 t a_{T}^{2} .
$$

Here we use bare in the sense of not including any effects of interactions with high-frequency modes which give rise to the pairing in our model.

For a Holstein model with anisotropy[73], the ratio of the transverse to longitudinal polaron masses is not much different from the same ratio for the bare masses, at least for interactions with high-frequency phonons. Thus, combining this result with the assumption that the ratio of the pair transverse mass $M_{T}$ to the bare transverse fermion mass $m_{F T}$ is approximately equal to the ratio of 
the pair longitudinal mass $M_{L}$ to the bare fermion longitudinal mass, which we take as equal to the free-electron mass $m_{e}$, we find

$$
M_{T} \approx 2 m_{F T b}\left(M_{L} / 2 m_{e}\right) .
$$

\section{Estimate of Energy of Mediating Bosons assuming these are Plas- mons}

We assume for most of this paper that the bosons mediating the attraction between fermions in the nanofilaments are plasmons, although we make some remarks in the discussion section about the alternative that they are highfrequency phonons.

As in Ref. [51, we estimate the plasmon energy as that for a single nanofil-

ament at wave numbers $q$ such that $q d \gtrsim 1$, where $d$ is the diameter of the nanofilament. In this case the plasmon energy is

$$
\hbar \omega=\hbar\left(16 \pi e^{2} c_{B} d^{-2} / M_{L} \epsilon_{h}\right)^{1 / 2},
$$

where $c_{B}$ is the linear boson concentration and $\epsilon_{h}$ is the high-frequency dielectric constant. The difference from the usual factor of $4 \pi$ in the bracket comes about since the pair charge is $2 e$. For the diameter $d$ in Eq. (31), we first estimate the mean value of the radius from the axis from Grigorov's variational method[48] as

$$
r_{m}=\int_{0}^{\infty} r^{2}(1+\beta r)^{2} e^{-2 \beta r} / \int_{0}^{\infty} r(1+\beta r)^{2} e^{-2 \beta r}=(14 / 9) \beta^{-1},
$$

where, from Eq. (24), $\beta=19.6\left(c_{B} a_{T}\right) / a_{T}$. For a uniform distribution of charge up to a cylinder radius of $r_{c}$, the mean value $r_{m}$ of $r$ is given by

$$
r_{m}=(2 / 3) r_{c} .
$$

Thus to convert the mean value of $r$ given above to a total cross section, we multiply the cross section by $(9 / 4)$, or the linear dimensions by $(3 / 2)$. Hence the diameter of an equivalent square filament is taken as

$$
d=(3 / 2)\left(14 \pi^{1 / 2} / 9 \beta\right)=0.211 a_{T} /\left(c_{B} a_{T}\right) .
$$

Hence, from Eq. (31),

$$
\hbar \omega \approx \hbar\left(16 \pi e^{2} c_{B} a_{T} / \epsilon_{h} M_{L} a_{T}^{3}\right)^{1 / 2}\left(4.74 c_{B} a_{T}\right) .
$$

There will be additional terms proportional to the square of the wave number and inversely proportional to the mass at high $q$, but, for parameters of interest, these are not expected to change the relevant average plasmon energy by large amounts, and will be partially cancelled by the reduction in plasmon energy at small $q$. 


\section{Approximate Relation between the Bipolaronic Binding Energy and the Bipolaron Mass}

We obtain another approximate relation between parameters by making use of results for bipolaron binding energies and masses for weak to intermediate coupling in a one-dimensional system[53]. Although these results were obtained with symmetric spatial bipolaron wave functions, implying singlet bipolarons, results for singlet and triplet bipolarons in a Holstein-Hubbard model do not look drastically different from each other for weak to intermediate coupling (see Figs. 1 and 2 of Ref. [74] or Fig. 15 of Ref. [75]). From section 4 of Ref. [53], for $V^{2}=0.125,0.25$ and 0.375 and $a=4$ in the notation of that paper, $a=4$ corresponding to high frequencies of the bosons mediating the electron-electron attraction, we can see that the ratio $\left(E_{b} / \hbar \omega\right) /\left[\left(M_{b p} / 2 m_{b}\right)-1\right]$ lies between 1.63 and 1.32, and, with extrapolation to higher $V^{2} a$ up to 0.5 , this value could go down to about 1.25. For values of $V^{2}$ between 0.125 and 0.375 , the values of the bipolaron binding energies $E_{p b}$ lie between $0.08 \hbar \omega$ and $0.38 \hbar \omega$, while the bipolaron masses $M_{L}$ (for a bare mass $m_{b}$ ) lie between 2.13 and $2.5 m_{b}$ for the same parameter range. Based on these results we shall suppose that

$$
M_{L} / m_{b}=2.0+(1.4 \pm 0.2)\left(E_{p b} / \hbar \omega\right),
$$

and in our case we suppose $m_{b}=m_{e}$, the free-electron mass.

$V$ and $a$ in our 2007 model, which uses local electron-boson interactions but a quadratic dispersion for bare electrons, correspond approximately to what are usually called $g$ and $(\hbar \omega / t)^{1 / 2}$ in the Holstein model. Thus $0.5 V^{2} a^{2}$ corresponds to $g^{2} \hbar \omega / 2 t$, usually called $\lambda$ in the 1-D Holstein model. Hence $V^{2} a=0.5$ and $a=4$ imply $\lambda \approx 2$. Fig. 1 of a paper by Hohenadler and von der Linden[76] shows that the kinetic energy (related to reciprocal masses) of Holstein bipolarons is reduced by a factor of more than five at $\lambda=2$. However, we expect reductions of masses in our model compared with those on the Holstein model to become larger as the coupling increases, and so the comparatively small masses for the values of $V^{2}$ and $a$ with which we have been concerned may be realistic for our model.

\section{Probable Lower and Upper Limits for the Bipolaron Binding En-} ergy

Although there are criteria for being on the BEC side of the BEC to BCS transition in terms of the product of the scattering length and the Fermi wave vector 77, 78, 79 or in terms of the ratio of $E_{p b}$ to $E_{F}$ (deducible from Fig. 1 of Ref. [80]), we are not sure how these are modified for systems which are both polarised and in which there is also a cut off in wave vector in two directions, with the Fermi wave vector having a value which is approximately equal to its 1 -D value. (How good this approximation is will be discussed after numerical work determined approximate values of parameters).

The simplest criterion for being on the BEC side of the transition is that the diameter of the pairs is smaller than their mean separation. In the longitudinal direction, the diameter of the pairs is approximately $\left(8 \hbar^{2} / M_{L} E_{p b}\right)^{1 / 2}$, 
assuming $M_{L} \approx$ (twice the longitudinal fermion mass), and the pair separation is approximately $\left(c_{B} a_{T}\right)^{-1} a_{T}$. Hence a lower limit for the pair binding energy is

$$
E_{p b}=16\left(\hbar^{2} / 2 M_{L} a_{T}^{2}\right)\left(c_{B} a_{T}\right)^{2} .
$$

Another lower limit on the pair binding energy requires that $E_{p b}$ is appreciably larger than $3.5 k_{B} \times 295 \mathrm{~K} \approx 0.08 \mathrm{eV}$ (BCS value of energy gap for $\left.T_{c}=295 \mathrm{~K}\right)$. We use the first of these limits, but after numerical work we also check that the value found is also above the second lower limit.

For an upper limit, based on our discussion in the previous section, and the assumption that we do not wish to venture far out of the intermediate-coupling régime, we suppose that the pair binding energy $E_{p b}$ satisfies $E_{p b}<0.6 \hbar \omega$. Hence, using Eq. (37), we put

$$
E_{p b} \sim 0.5\left[16 \hbar^{2} /\left(2 M_{L} a_{T}^{2}\right)\left(c_{B} a_{T}\right)^{2}+0.6 \hbar \omega\right] .
$$

\section{Order of Magnitude Constraint from Critical Current Density}

Estimates of the critical current density made from pulsed-current measurements vary from 81$] 10^{8} \mathrm{Acm}^{-2}$ to 5$] 5 \times 10^{9} \mathrm{Acm}^{-2}$, depending on whether the size of the pressure contacts or the estimated cross section of a single channel is used to convert a critical current of $(63 \pm 17) \mathrm{A}$, for gradually increasing magnitude of the currents in the pulses, to a current density. We take the geometric mean of these two types of estimates, i.e.

$$
j_{c} \sim 7 \times 10^{8} \mathrm{Acm}^{-2} .
$$

In numerical studies of a fermion superfluid, Heiselberg 82 shows that, for the Bose-gas regime, the sound velocity at low temperatures (equal to the critical drift velocity $v_{d}$ for a Bose gas) lies between about 0.08 and 0.25 times the Fermi velocity, $v_{F}$. Thus we assume that

$$
v_{d}=(0.165 \pm 0.085) v_{F} .
$$

Since the critical temperature in the pulsed currents used is estimated in Ref. [5] to be greater than $700 \mathrm{~K}$, we assume that room temperature will count as a fairly low temperature for the purposes of estimating the critical current. Hence, using Eqs. (39) and (40), we find for our model that

$$
(0.33 \pm 0.17) e\left(c_{B} a_{T}\right) a_{T}^{-3} v_{F} \sim 7 \times 10^{8} \mathrm{Acm}^{-2}=2.1 \times 10^{18} \mathrm{esu} .
$$

We approximate the Fermi velocity by that for a one-dimensional ferromagnetic system as in the previous section. Then we find

$$
v_{F} \approx \hbar(2 \pi) c_{B} / m_{F L} \approx \hbar(4 \pi)\left(c_{B} a_{T}\right) / a_{T} M_{L} .
$$

Eqs. (41) and (42) give another order-of-magnitude relation between parameters of our model. 


\section{Estimate of Effective Pair Chemical Potential}

We estimate an effective pair chemical potential to put into the Bogoliubovtype of dispersion for non-interacting bosons at $T_{c}$ as follows. First we look at accurate results for the condensation temperature for interacting bosons 56, 57. at low carrier concentrations, where it is found that the change $\Delta T_{c}$ from the non-interacting boson value $T_{c 0}$ is given by

$$
\Delta T_{c} / T_{c 0} \approx 1.3 a_{B} n_{B}^{1 / 3},
$$

where $a_{B}$ is the boson scattering length. Equation (43) is only valid for low boson concentrations, but decreases of $T_{c}$ due to interactions for higher concentrations [58] can be taken into account in a non-interacting boson model by assuming an appropriate increase in mass with $n_{B}$ with a dependence on density with a higher power of $n_{B}$ than $1 / 3$.

The boson scattering length in Eq. (43) satisfies

$$
a_{B} \approx 0.6 a_{F},
$$

where $a_{F}$ is the fermion scattering length $[83$, which in turn is related to the pair binding energy $E_{p b}$ and fermion mass $m_{F}$ by 84

$$
\hbar^{2} /\left(m_{F} a_{F}^{2}\right)=E_{p b} .
$$

Assuming the fermion mass is approximately equal to half the boson mass $M_{B}$, we deduce from Eqs. (44) and (45) that

$$
a_{B} \approx 0.6\left(4 \hbar^{2} / 2 M_{B} E_{p b}\right)^{1 / 2} .
$$

For an anisotropic system we suppose that $a_{B}$ to use in (43) is as in (46), but with

$$
1 / M_{B}^{1 / 2} \rightarrow(1 / 3)\left(1+2 p^{1 / 2}\right) / M_{L}^{1 / 2}
$$

in our notation. Also, in our system,

$$
n_{B}^{1 / 3}=\left(c_{B} a_{T}\right)^{1 / 3} a_{T}^{-1} .
$$

Hence, from Eqs. (43) to (48) we find

$$
\Delta T_{c} / T_{c 0} \approx 0.52\left(\hbar^{2} / 2 M_{L} E_{b} a_{T}^{2}\right)^{1 / 2}\left(1+2 p^{1 / 2}\right)\left(c_{B} a_{T}\right)^{1 / 3}
$$

For non-interacting bosons with a Bogoliubov type of dispersion at $T_{c}$, we find numerically the $\mu_{B}$ which gives the same fractional change of $T_{c}$ from that for a pure quadratic dispersion as for the interacting system as determined above. Hence we find $\mu_{B}$ as a function of our other parameters.

12. Change in Carrier Concentration in $0.3 \mu \mathrm{m}$ Films required to make the $T_{c}$ of the Highest-Resistance Superconducting Channel for these Films equal to Room Temperature 
Now, because of possible injection of carriers from the electrodes (see the Introduction), we assume that

$$
c_{B} a_{T} \rightarrow c_{B} a_{T}+\Delta\left(c_{B} a_{T}\right)=c_{B 2} a_{T} \text { (say) }
$$

for $0.3 \mu \mathrm{m}$ films. Then $p$ in our equations in section 2 has to be replaced by $p_{2}=M_{L} / M_{T 2}$, where the transverse mass $M_{T 2}$ for the thinner films is calculated as in section 6 , but with $c_{B} a_{T}$ replaced by $c_{B 2} a_{T}$.

For a fixed boson concentration, the decrease $\Delta T$ of $T_{c}$ is taken to be given by $\Delta T \propto n_{T}^{-4 / 3}$ [see Eq. (11)], but we also suppose that $\Delta T$ is proportional to the inverse of the carrier concentration in order to take into account that phase slips are not so easily formed if the carrier concentration rises. Hence we suppose that the reduction in $T_{c}, \Delta T_{2}$, for the highest resistance superconducting channel in the $0.3 \mu \mathrm{m}$ films, with $n_{T}=17$, is related to the corresponding quantity, $\Delta T_{1}$ for $50 \mu \mathrm{m}$ films, with $n_{T}=32$, by

$$
\Delta T_{2} \approx 2.3\left(c_{B} a_{T} / c_{B 2} a_{T}\right) \Delta T_{1},
$$

where $c_{B 2}$ is the linear carrier concentration in the $0.3 \mu \mathrm{m}$ films.

After other parameters are determined, $c_{B 2} a_{T}$ is varied so that

$$
T_{B}-\Delta T_{2}=295 K
$$

for $n_{T}=17$. The change in $c_{B} a_{T}$ also gives rise to a change in $p=M_{L} / M_{T}$ because it changes the potential involved in calculating the transverse mass.

\section{Numerical Results}

For our numerical work we first choose a value for the parameter $\Delta T_{1} . T_{B}$ in our model as a function of $n_{T}$ reaches a shallow maximum as a function of $n_{T}$, and then declines slightly as $n_{T}$ decreases further. (A qualitative discussion of why this slight decline occurs has been given for bosons with a quadratic dispersion in Ref. [63]). However, the results shown in Fig. 1 indicate that once $T_{c}$ reaches room temperature it remains above that temperature as $n_{T}$ increases further, at least up to very high $n_{T}$. Hence, when other parameters are approximately determined, we may obtain a lower limit for $\Delta T_{1}$ from this condition. For our final values of parameters we find that $\Delta T_{1}>7 K$, and for our calculations we take a value

$$
\Delta T_{1}=15 \mathrm{~K}
$$

That $\Delta T_{1}$ is probably not much larger than this may be argued by comparison with conclusions of Zhao for carbon nanotubes [8, 9]. He gives an example (see pp 46-48 of Ref. [9]) where phase slips give rise to a resistance equal to about $0.4 \times$ (the normal-state resistance at a temperature of the order of $0.8 \times$ the transition temperature without phase slips) for a number of (spin-degenerate) channels equal to 54 , corresponding to $N_{T}^{2}=108$ for our type of system, where we suppose there is no spin degeneracy. Hence, if we assume that Eq. (11) is true, for our case we might suppose that $\Delta T_{1} / T_{B}$ for $32^{2}=1024$ nanofilaments 
is slightly larger than $\left[(1024 / 108)^{-2 / 3}=0.223\right] \times 0.2 \approx 0.045$, implying $\Delta T_{1}$, given by $\Delta T_{1}=0.045 \times\left(295 \mathrm{~K}+\Delta T_{1}\right)$ is slightly larger than $14 \mathrm{~K}$. Of course this is only an order-of-magnitude estimate because Eq. (11) and the analysis of Zhao are based on a theory appropriate for materials on the BCS side of the BCS-BEC transition, whereas we are assuming we are on the opposite side.

Having determined $n_{T}$ for the highest-resistance superconducting channels in section 3 , and chosen the value of $\Delta T_{1}$ given by Eq. (53), we next make use of the requirement that $T_{c} \approx 295 \mathrm{~K}$ for the highest-resistance superconducting channels for $50 \mu \mathrm{m}$ films and results of sections 4 to 11 to determine another eight parameters of our theory approximately. These are $a_{T}, c_{B} a_{T}$ for $50 \mu \mathrm{m}$ films, $a_{T} / \rho, E_{b}, M_{L}, p=M_{L} / M_{T}$, $\hbar \omega$ and $\mu_{B}$. This gives nine approximate equations for eight parameters, and so one might think that the parameters are overdetermined. However, because many of the relations between parameters are very approximate, we do not have to worry about satisfying them exactly, and it turns out that we can satisfy them to within our estimated accuracy for the equations.

Our procedure is as follows. We regard Eq. (16) of section 4 as exact, and then minimise a weighted sum of moduli of differences between sides of dimensionless quantities based on other equations, with weightings based on our estimates of accuracy of our equations. In order not to cause computing time to be too long, we approximate the sums required to produce $T_{B}$ given in section 2 by grouping terms in the sums over $k$ in section 2 as equal to their mean values between two limits, the differences between these limits increasing as $k$ increases. For our detailed calculations, we regard the numerical equation giving $T_{c}=295 \mathrm{~K}$ for the highest-resistance superconducting channel for $50 \mu \mathrm{m}$ films as accurate to less than $1 \%$, but supplemented by errors of up to about $1 \%$ because of our approximate methods of evaluating the sums over $k$ in equations of section 2. We weight the differences in moduli of calculated and fitted values of quantities $x$ divided by the fitted values, i.e.

$$
\left|\left(x_{c}-x\right) / x\right|
$$

by weightings according to our guesses of the accuracy of the equations. Taking the weighting for $\left|\left[\left(T_{c c}-295 K\right) / 295 K\right]\right|$, where $T_{c c}$ is the calculated $T_{c}$ for the highest-resistance superconducting channel for $50 \mu \mathrm{m}$ films, as 1 , we choose the following much smaller weightings for similar quantities associated with $a_{T}$ given by Eq. (21) (0.06), $j_{c}$ given by Eqs. (41) and (42) (0.015), $p=M_{L} / M_{T}$ given by the methods described in section $6(0.03), \hbar \omega$ based on Eq. (35) (0.1), $E_{p b}$ based on Eq. (38) (0.03), $M_{L}$ based on Eq. (36) (0.2), and $\mu_{B}$ based on methods described in section $11(0.1)$. There is some guessing as to the appropriate weightings. The weighting for the transverse bipolaron mass is taken as fairly low because its calculation depends both on details of Grigorov's variational method, and assumptions about how to convert the masses deduced from his theory to masses for pairs. The very small weighting for $j_{c}$ is due to the uncertainty in the experimental values of critical currents, the larger uncertainty arising from conversion to a critical current density, the uncertainty to what 
extent $295 \mathrm{~K}$ represents a "low $T$ " for calculation of critical currents, and the uncertainty about how much other parameters change when large currents are passed. The weighting for $E_{p b}$ is small because for typical values of parameters the ratio of the upper and lower limits is quite large. For computational purposes, for calculations of $p$ we approximated results for logarithms of expressions as polynomials in relevant parameters, and for $\mu_{B}$ we approximated by a different simple expression in the relevant parameters, and used the polynomial forms or other simple expressions in our minimisation programs. Since $a_{T} / \rho>2$, but probably not much greater, we only did calculations for $c_{B} a_{T} \quad\left(=0.259 a_{T} / \rho\right)$ between 0.5 and 0.7. For the eight-parameter minimisation, we made use of a program AMOEBA from a book [85], based on a method suggested by Nelder and Mead 86 . For our computing we used dimensionless lengths in units of $\mathrm{nm}$, dimensionless masses in units of $m_{e}$, and dimensionless energies in units of $\left(\hbar^{2} / 2 M_{L}\right)\left(\pi / a_{T}\right)^{2}$.

From the best fit to the weighted sum of moduli as given by Eq. (54), we find that

$$
T_{c}=295.03 \mathrm{~K}, a_{T} / \rho=2.061,
$$

implying with Eq. (16) that $c_{B} a_{T}=0.534$, and fitted and calculated values $x$ and $x_{c}$ of other quantities, and of $\left[\left(x_{c}-x\right) / x\right]$ found for (i) $a_{T}$ from Eq. (21), (ii) $p$ from section 6, (iii) the plasmon energy from Eq. (35), (iv) $M_{L}$ from Eq. (36), $E_{p b}$ from Eq. (38), (v) $j_{c}$ from Eqs. (41) and (42), (vi) $\mu_{B}$ from section 11 are:

$$
\begin{array}{rc}
a_{T}=1.282 \mathrm{~nm}, & \left(a_{T c}-a_{T}\right) / a_{T}=0.033 \\
p=0.315, & \left(p_{c}-p\right) / p=0.002 \\
\hbar \omega=1.31 \mathrm{eV} & \left(\hbar \omega_{c}-\hbar \omega\right) / \hbar \omega=-1.3 \times 10^{-6} \\
M_{L}=2.376 m_{e}, & \left(M_{L c}-M_{L}\right) / M_{L}=0.029 \\
E_{p b}=0.415 \mathrm{eV}, & \left(E_{p b c}-E_{p b}\right) / E_{p b}=7 \times 10^{-5} \\
j_{c}=3.43 \times 10^{8} \mathrm{Acm}^{-2}, & \left(j_{c c}-j_{c}\right) / j_{c}=-0.510 \\
\mu_{B}=0.00127 \mathrm{eV}, & \left(\mu_{B c}-\mu_{B}\right) / \mu_{B}=1.1 \times 10^{-5} .
\end{array}
$$

The only quantity for which the calculated values and the values obtained by our fitting process is significantly different is $j_{c}$. Some reasons why we expected that the $j_{c}$ estimate would be inaccurate are stated earlier in the section. For the values of parameters found, the upper limit for $E_{p b}$ in section 9 is $0.79 \mathrm{eV}$ and the lower limit is $0.045 \mathrm{eV}$. It turns out that our lower limit is smaller than the alternative lower limit of $0.08 \mathrm{eV}$ given in section 9 , but if we had used this alternative in our computation we do not expect that there would be much change in parameter values.

Note that the small differences in most cases between calculated and fitted parameters shown above do not imply that the parameters are known to anything like that accuracy, as errors cannot be expected to be less than our estimated errors in the calculated relations used in our fitting process.

After the other parameters are obtained, we treat $c_{B 2} a_{T}$ for the channels through $0.3 \mu \mathrm{m}$ films as adjustable, where $c_{B 2}$ is the linear concentration of 
bosons in these channels. The calculation of transverse mass in this calculation has to be modified for the different carrier concentration. We find that $c_{B 2} a_{T}=$ 0.636 (compared with $c_{B} a_{T}=0.534$ for $50 \mu \mathrm{m}$ films), and the the ratio $p_{2}$ of longitudiinal to transverse mass in the channels through the thinner films is given by $p_{2}=0.258$, smaller than the value $p=0.315$ for the thicker films. Note that, if other parameters were to remain fixed, then $T_{B}$ would increase with boson concentration. However, $p=M_{L} / M_{T}$ decreases with increase of $c_{B}$ because of the increase in depth of the potential of Eq. (25) with increasing $c_{B}$. Thus there is a subtle competition between two effects in the parameter range in which we are interested.

For the values of parameters found, $T_{c}=T_{B}-15 \mathrm{~K} \times\left(n_{T} / 32\right)^{-4 / 3}$ reaches a maximum of about $303 \mathrm{~K}$ for $50 \mu \mathrm{m}$ films for $n_{T} \approx 140$, and $T_{c}=T_{B}-15$ $\mathrm{K} \times\left(n_{T} / 32\right)^{-4 / 3}\left(c_{B} a_{T} / c_{B 2} a_{T}\right)$ for $0.3 \mu \mathrm{m}$ films reaches a maximum of about $310 \mathrm{~K}$ for $n_{T} \approx 90$, assuming channels are single domains with all spins aligned. For relatively large channels it is likely that the channels will split up into domains with opposite directions of spins, but we do not know enough about the transverse exchange constants or magnetic anisotropy to be able to predict the maximum diameter of single-domain channels. In order to explain higher $T_{c}$ 's than the two values noted above, observed when relatively high currents are applied[2, we can invoke decreases in transverse masses due to reduced lattice constants produced by current-current interactions between nanofilaments as discussed in some detail in Ref. 3].

\section{Discussion}

In this section we discuss many of the assumptions that come into our model, and to what extent they can be justified. We also discuss some other matters.

Assumption of an array of nanofilaments

There is at present no experimental evidence that the conducting channels are composed of an array of nanofilaments, and so for this assumption we have to rely on the plausibility of the theory of Refs. [46, 47, 48, for strings of an unusual type of polaron occurring in elastomers with rotatable dipolar groups. In this connection we remark that there are also theories for strings of more conventional polarons (Ref. [87] and references therein, also Ref. [88]), although to form these one has to include interactions other than the usual Fröhlich interactions with polar phonons and Coulomb repulsions, and there are limits to the lengths of the strings 88 .

\section{Assumption of a square lattice}

We cannot rule out a triangular lattice. Our use of a square lattice is based on the simplicity of calculations, and the probability that results for a triangular lattice will not be much different, at least for weak or intermediate coupling of electrons and bosons mediating the attraction. For strong coupling a triangu- 
lar lattice is likely to be favoured because of the smaller transverse masses of bipolarons on such a lattice when coupling becomes strong [89].

Assumption that our system is on the BEC side of the BEC-BCS transition We estimate the pair diameter $d_{p}$ in the longitudinal direction from the pair binding energy and reduced mass of the particles making up the pair, and compare this with the separation between pairs $s \approx\left(c_{B} a_{T}\right)^{-1} a_{T}$. We use the approximation sign rather than the equality for $s$ because there will be a slight deviation from this value towards the (lower) average separation $\left(c_{B} a_{T}\right)^{-1 / 3} a_{T}$ which would occur if the system were three-dimensional with no cut off in wave vector in the transverse direction. We find $d_{p}=4\left(\hbar^{2} / 2 M_{L} E_{p b}\right)^{1 / 2}=0.79 \mathrm{~nm}$, $s \approx 2.40 \mathrm{~nm}$, and so the diameter of the pairs is considerably smaller than their mean separation. This shows that we are well on the BEC side of the transition.

\section{Assumption of good contacts}

If the contacts were not good, then $n_{T}$ for the highest-resistance superconducting channels would have to be larger for both thicknesses of films, and then it would be more difficult to justify a $\Delta T_{1}$ as large as we need to permit $T_{c}$ to increase monotonically with $n_{T}$. Also, from Fig. 1, the ratio of resistances of the lowest-resistance mediumly conducting channels imply, taking into account the different channel lengths, that the ratios of cross sections for the lowestresistance normal channels for the two film thicknesses needs to be about four, the same as inferred for the highest-resistance superconducting channels assuming good contacts. Hence, if the contacts are not good, they would have to have about the same departure from goodness for both thicknesses of films, which would be another assumption. For discussion of normal channel resistances, although with smaller values of $n_{T}$ than given by the present model, see Ref. [3.

Assumption of a Bogoliubov-type of dispersion for bosons at $T_{c}$ A possible justification for this assumption is discussed in the Introduction. We tried to fit the data assuming a simple quadratic dispersion, but were unable to do this with plausible values of parameters. However, we could fit the data with a fixed value of $\mu_{B}$ [in units of $E_{u}=\left(\hbar^{2} / 2 M_{L}\right)\left(\pi / a_{T}\right)^{2}$ ] over three orders of magnitude smaller than that found by our data fitting. For $\mu_{B} / E_{u}=10^{-5}$ [cf. $\mu_{B} / E_{u}=0.0132$ for the results given in section 13], $a_{T}$ is reduced to 1.037 $\mathrm{nm}, p$ is increased to $0.423, M_{L}=2.444 m_{e}$, and $a_{T} / \rho=2.000$. For $\mu_{B} / E_{u}$ significantly smaller, $T_{B}$ for $n_{T}=32$ is too small for plausible values of $a_{T}$ and boson concentrations, e.g. for $\mu_{B}=0, L=50 \mu \mathrm{m}, a_{T}=1 \mathrm{~nm}, p=0.5, c a_{T}=$ $0.54, M_{L}=2.55 m_{e}, T_{B}$ for $n_{T}=32$ is only $25 \mathrm{~K}$, compared with nearly $500 \mathrm{~K}$ for large $n_{T}$. The only possibility to fit data with $\mu_{B}=0$ would be to assume poor contacts, so that the lowest $n_{T}$ to give $T_{c}=295 \mathrm{~K}$ is considerably larger. We tried fitting assuming that $n_{T}$ for the highest-resistance superconducting 
channel for the $50 \mu \mathrm{m}$ films was $n_{T}=100$ (setting $\Delta T=0$ in this case because of the large $n_{T}$ ), but did not get nearly so good a fit as for our Bogoliubov-type dispersion and $n_{T}=32$. For $n_{T}=100$, we found that the calculated value of $a_{T}$ was $51 \%$ higher than the fitted value, the calculated value of $p$ was $72 \%$ lower than the fitted value, and the weighted sum of moduli of differences between calculated and fitted values was about seven times higher than for our previously reported results for $n_{T}=32$ and $\mu_{B}$ as a fitting parameter. The problem with trying to fit with low values of $n_{T}$ for $\mu_{B}=0$ is that, for quadratic dispersion, the rise of $T_{B}$ with $n_{T}$ is rather slow, and so, if we use Grigorov's expressions for linear carrier concentrations $c_{B}$, it is not possible to get $T_{B}$ for small $n_{T}$ up to room temperature unless $a_{T}$ is small, and then it becomes impossible to satify Eq. (21), since we have the restriction that $a_{T} / \rho>2$. Another problem is the strong length dependence of $T_{B}$ for quadratic dispersion (proportional to $1 / L$ for small $n_{T}$ ), and so one would need to have a considerable change in carrier concentration in the $0.3 \mu \mathrm{m}$ films to find a room-temperature $T_{B}$ for the highest-resistance superconducting channel with parameters determined by fitting $T_{B}$ for the highest-resistance superconducting channel for $50 \mu \mathrm{m}$ films. Since computation time for our program goes up as the square of $n_{T}$, it is not practicable to see whether things improve for pure quadratic dispersion if $n_{T}$ is taken as much larger than 100, without making further approximations for $T_{B}$. For the rather drastic approximation made by Eq. (21) of Ref. 63, but increased in magnitude by $15 \%$, since, for the cases we studied there gave errors of the order of $15 \%$ in $T_{B}$, we found that the best fit was obtained when $n_{T}$ for the highest-resistance superconducting channel for the $50 \mu \mathrm{m}$ film was about 240 , with values of other parameters as follows:

$p=0.596, a_{T} / \rho=2.003, a_{T}=0.942 \mathrm{~nm}, M_{L} / m_{e}=2.444, \hbar \omega=1.96 \mathrm{eV}$, $E_{b}=0.621 \mathrm{eV}, j_{c}=1.08 \times 10^{9} \mathrm{~A} \mathrm{~cm}^{-2}$. However, neither the goodness of the fit nor values of all parameters vary monotonically with $n_{T}$, which may indicate that we do not always reach the absolute minimum of our weighted sums with our program. For these values of parameters, $n_{T} a_{T}$ for the highest resistance superconducting channel for $50 \mu \mathrm{m}$ films is approximately equal to $230 \mathrm{~nm}$. Since there is a spread of resistance for the low-resistance channels for these films shown in Fig. 1 of about 100, this would imply that the widest channels shown in Fig. 1 would be about $2.3 \mu \mathrm{m}$ in diameter, slightly larger than the largest possible value mentioned in Ref. 5 .

We may look at results for pure quadratic dispersion in more detail sometime, if we can find a better approximation to $T_{B}$ which does not require too much computing time to investigate. Although we think that our arguments about fluctuations giving parts of the samples at any one time with higher $T_{B}$ 's than average, and hence a Bogoliubov-type of dispersion for these parts is plausible, it is less clear whether it is appropriate to consider some average type of Bogoliubov dispersion over the whole sample with a lower effective value of $\mu_{B}$, as we have done for most of this paper.

Remarks on Grigorov's theory for ferromagnetic nanofilaments 
It might be objected that a paper by Lieb and Mattis 90 could rule out ferromagnetism in quasi 1-D filaments. However, on studying this paper, it appeared to the present author that Lieb and Mattis's result depended on the system being really one dimensional, not just quasi one dimensional in the sense that all the carriers are in the lowest state with respect to transverse motion, and thus we think their result is not relevant to any real system. Grigorov[48] also notes that the result of Lieb and Mattis is based on a model with short-range forces. Further, Shelykh et al.71] also find ferromagnetism in a quasi one-dimensional electron gas, and Meyer and Matveev [91] mention, in connection with quantum wires, that "deviation from true one dimensionality may, in principle, give rise to a spin-polarised ground state of the interacting electron system".

There are more general objections to any form of long range order in quasi one-dimensional systems, but these will not be relevant for arrays of nanofilaments.

\section{Stability of electron strings}

Grigorov 48 finds that the ferromagnetic electron string is only stable if $R_{0}$, the radius from an excess electron up to which all dipoles point away from the electron, satisfies $R_{0}>0.6 \mathrm{~nm}$. For our parameters and equations in section 4 we find $R_{0} \approx 0.61 \mathrm{~nm}$. Putting this value into Grigorov's Eq. (9), with the second term reduced by a factor of 0.8 since we are assuming $k=\epsilon_{h} / \epsilon_{s}=0.8$ instead of unity as in his calculations, and using $\epsilon_{h}=2.2$, we find $E_{\text {min }}=(0.96-0.61)=$ $0.35 \mathrm{eV}$. However, in our model there is a reduction in energy due to polaron and bipolaron binding energies caused by interaction with high-energy bosons. From our fitting we find that the bipolaron binding energy $E_{p b} \approx 0.42 \mathrm{eV}$, with $E_{p b} / \hbar \omega=0.32$. From Ref. [53], section 4, for $a=4$, corresponding to $\hbar \omega / t=16$, this value of $E_{p b} / \hbar \omega$ probably corresponds to $V^{2} \approx 0.29$. Then for weak coupling and $a=4$, from equation (46) of Ref. [53, the single-polaron binding energy $E_{s b}=0.28 \hbar \omega=0.37 \mathrm{eV}$. Hence we get a total energy lowering per electron of $E_{s b}+0.5 E_{p b}=0.58 \mathrm{eV}$, quite enough to stabilise the system.

Note that the value of $R_{0}=0.61 \mathrm{~nm}$ noted above that we find is considerably smaller than the estimate that $R_{0}$ is greater than about $2 \mathrm{~nm}$ mentioned in Ref. [48, and so, if our parameters are approximately correct, then we think Grigorov must have underestimated the shear modulus $G$ in Eq. (12). Other quantities in this equation are probably known far better than $G$.

\section{Alternative assumption for $\hbar \omega$}

There are high-frequency phonons in polypropylene with energies $\hbar \omega=0.37-$ $0.39 \mathrm{eV}[92$. We have also looked at our model on the assumption that phonons of energy $0.38 \mathrm{eV}$ mediate the attraction. The best fit when minimising the weighted sum of the same quantities as before except for the calculated and fitted frequency of bosons, gives $a_{T} / \rho=2.055$, and the following values of other parameters and departures from their calculated values:

$$
a_{T}=1.322 \mathrm{~nm}, \quad\left(a_{T c}-a_{T}\right) / a_{T}=-8 \times 10^{-7}
$$




$$
\begin{array}{rc}
p=0.234, & \left(p_{c}-p\right) / p=9 \times 10^{-7} \\
M_{L}=2.527 m_{e} & \left(M_{L c}-M_{L}\right) / M_{L}=-7 \times 10^{-4} \\
E_{p b}=0.143 \mathrm{eV}, & \left(E_{p b c}-E_{p b}\right) / E_{p b}=-0.063 \\
j_{c}=2.84 \times 10^{8} \mathrm{Acm}^{-2} & \left(j_{c c}-j_{c}\right) / j_{c}=-0.595 \\
\mu_{B}=0.00263 \mathrm{eV}, & \left(\mu_{B c}-\mu_{B}\right) / \mu_{B}=1.1 \times 10^{-7} .
\end{array}
$$

Differences of calculated and fitted parameters are on average not much different from values obtained assuming plasmons mediate the interaction. For these parameters we find $d_{p}=1.29 \mathrm{~nm}$ and $s \approx 2.48 \mathrm{~nm}$, and so we are still on the BEC side of the transition, although not quite so far on this side as for our earlier set of parameters.

Alternative theory of Grigorov for superconductivity

Grigorov [4, 50] has a completely different type of theory for superconducting channels involving pairs of oppositely moving charged density waves along each superpolaron string. In his theory he appears to think that the superconductivity will occur even for isolated superpolaron strings. If that were the case we would have to look for a different explanation for the difference between normal and superconducting channels from that used here, and for the difference in resistances of the highest-resistance superconducting channels for the two film thicknesses.

Remarks on use of one-dimensional Fermi wave vector

Our model does not involve a truly one-dimensional system. However, for our fitted values of parameters, the transverse wave vector for a given longitudinal wave number $k_{L}$ reaches its maximum possible value $\pi / a_{T}$ for $k_{L}$ up to a value equal to a large fraction of the calculated 1-D Fermi wave vector for a ferromagnetic system of $k_{F, 1-D}=\pi c_{F}=2 \pi c_{B}$, where $c_{F}$ and $c_{B}$ are the linear concentrations of fermions and bosons. Bearing this in mind, the longitudinal Fermi wave vector $k_{F L}$ can be estimated as follows. First we approximate the square projection of the Brillouin zone on the $x y$-plane by a circle of radius $k_{c}$ with the same area, i.e. of radius given by $\pi k_{c}^{2}=\left(2 \pi / a_{T}\right)^{2}$, or

$$
k_{c}=2 \pi^{1 / 2} / a_{T} .
$$

Then the maximum value of $k_{m}$ of $k$ at which the transverse wave vector equals $\pi / a_{T}$ is given by

$$
k_{m}=\left(k_{F L}^{2}-p k_{c}^{2}\right)^{1 / 2} .
$$

If the longitudinal wave vector is $k_{F L}$, then the volume of the Brillouin zone is the same as that for an area $\pi k_{c}^{2}=4\left(\pi / a_{T}\right)^{2}$ and length $2\left[(1 / 4) k_{F L}+\right.$ $\left.(3 / 4) k_{m}\right]$. The factor of $3 / 4$ for the weighting of $k_{m}$ occurs because of the quadratic dependence of the relevant cross-sectional area on $\left(k_{F L}-k\right)$ up to $k=k_{m}$. Since the volume of the Brillouin zone $V_{B Z}=8 \pi^{3} n_{F}=16 \pi^{3} n_{B}$, 
where $n_{F}$ and $n_{B}$ are the concentrations of fermions and bosons, we deduce an equation for $k_{F L}$,

$$
2\left[(1 / 4) k_{F L}+(3 / 4)\left(k_{F L}^{2}-4 p \pi / a_{T}^{2}\right)^{1 / 2}\right]\left(2 \pi / a_{T}\right)^{2}=16 \pi^{3}\left(c_{B} a_{T}\right) / a_{T}^{3} .
$$

Putting in parameters from our model into these equations, we find that $k_{F, 1-D}=2 \pi\left(c_{B} a_{T}\right) / a_{T}=2.62 \mathrm{~nm}^{-1}$ and $k_{F L}=2.95 \mathrm{~nm}^{-1}$, i.e. the longitudinal Fermi wave vector is $13 \%$ larger than the 1-D value. This is an error comparable with or smaller than most of our estimated errors in calculated relations between various quantities in our theory. Note that, even if the longitudinal and transverse masses were equal, the value of the longitudinal Fermi wave vector would be only $37 \%$ larger than the 1 -D value for the same values of other parameters used, and so it is the cut off in transverse wave vectors rather than the smaller transverse mass which is the main contributor to the fact that the 1-D Fermi wave vector is a fair approximation.

Effect of replacing the probable tight-binding type of transverse dispersion by one with a constant mass

For a given mass at the bottom of a band, the single-particle bandwidth in the $x$ or $y$ direction is larger by a factor of $\pi^{2} / 4 \approx 2.5$ for a band with a constant bare mass than for a tight-binding band. For a pair band made up from singleparticle states with a tight-binding dispersion, it is less obvious what happens to the bandwidth. If we form pair states from two single-particle states with equal wave vectors (which may be a fair approximation for weakly bound pairs), but do not consider pair states with transverse wave vector greater than $\pi / a_{T}$ because of instabilities (cf. 69, 70]), then the width in the $x$ and $y$ directions due to a tight-binding model will only be decreased by a factor $\left(\pi^{2} / 8\right) \approx 1.23$. However, whatever happens to the pair bandwidth, if the condensation temperature is such that $k_{B} T_{B}$ is large compared with the transverse bandwidth, we expect the bandwidth is what is most important, whereas for the opposite inequality the states near the bottom of the transverse band are what matters. For our parameters the transverse bandwidth in the $x$ and $y$ directions is $p\left(\hbar^{2} / 2 M_{L}\right)\left(\pi / a_{T}\right)^{2}=0.0293 \mathrm{eV}$, and in the [110] direction twice this. Thus we expect that with a tight-binding model the transverse mass at the bottom of the band to fit the data would be somewhat smaller that determined by our data fitting, but probably not much smaller.

Value of $\mu_{B}$ at $T_{c}$ compared with $\mu_{B 0}$ at $T=0$.

The value of the sound velocity $v_{s}$ at $T=0$ in the Bogoliubov theory is

$$
v_{s}=\left(\mu_{B 0} / M_{B}\right)^{1 / 2}
$$

where $\mu_{B 0}$ is the bosonic chemical potential at $T=0$ and $M_{B}$ is the boson mass (see e.g. Ref. [84]). For the sound velocity along the channel direction we use the longitudinal boson mass $M_{L}$, and we also note that, in the BEC régime 82

$$
v_{s}=(0.165 \pm 0.085) v_{F}
$$


(see section 10), where the Fermi velocity in the longitudinal direction is given by

$$
v_{F L}=\hbar k_{F L} / m_{F L} \approx 2 \hbar k_{F L} / M_{L} .
$$

We deduce from Eqs. (61) to (63), with $M_{B}=M_{L}$ and $v_{F}=v_{F L}$ that

$$
\mu_{B 0} \approx 0.22\left(\hbar^{2} k_{F L}^{2} / 2 M_{L}\right) .
$$

Using the 1-D value for $k_{F L}$ and putting in values from section 13 for $M_{L}$, $c_{B} a_{T}=0.259\left(a_{T} / \rho\right)$ and $a_{T}$, we deduce that $\mu_{B 0} \approx 0.024 \mathrm{eV}$. Hence, with our value for $\mu_{B}$ at $T_{c}$ of $0.00127 \mathrm{eV}$, we find $\mu_{B} / \mu_{B 0} \approx 0.05$, with quite a large uncertainty because of the uncertainties in Eq. (62). Interpreting the above ratio as the fraction of fluctuating condensate at $T=T_{c}$, we deduce that this fraction is about $5 \%$. We do not have a theory for this fraction at present, and so cannot say whether its value is what should be expected.

Coincidence of maximum $T_{c}$ at low currents being only slightly above room temperature

Within our theory as presented, the only way to increase the maximum $T_{c}$ would be to increase $\Delta T_{1}$ to considerably higher values than the value of $15 \mathrm{~K}$ assumed, and adjusting other parameters accordingly. This would require ignoring our estimated order-of-magnitude value of $\Delta T_{1}$ based on comparison with analysis of Zhao for carbon nanotubes assumed to be in the BCS-régime.

\section{Shortage of experimental input}

Although we have managed to determine twelve parameters in our theory approximately, most of the information needed to determine them has come from theory rather than from experiment. The only use of experimental data has been (i) the use of the histograms in Fig. 1 to determine the probable value of the lowest $n_{T}$ for two film thicknesses for which the superconducting $T_{c}$ reaches room temperature, (ii) the very approximate estimate of carrier concentration in the channels used in section 5 , and (iii) the even more approximate estimate of the critical current density given in section 10. There is further information from magnetic properties which could be used in principle. However, we have had to abandon a theory for the metamagnetic transition given in Ref. 52 because recent work 53 , on bipolaron dispersion at intermediate coupling in one dimension does not support the theory of great enhancements of electronelectron attractions at high drift velocities [51] on which the theory was based. The large diamagnetism in some samples has been interpreted in terms of induced currents in superconducting channels forming closed loops 21, but this interpretation does not help to determine any of our parameters, except to give an alternative estimate of channel diameters to those given in [5]. In Ref. 21] we estimated that diameters for channels which formed closed loops in one sample must be greater than $1.52 \mu \mathrm{m}$. 
Some suggested experiments

The greatest need in this field is for more experimental groups to try to reproduce all experimental results previously reported mainly by one group, at least as far as claims of room-temperature superconductivity are concerned, and to perform various new experiments. Nine suggestions for experiments were given in section 3 of Ref. [52. Two of these were mainly to test a model for metamagnetism given there which we no longer believe, and another suggestion may not be practicable, but most of the others seem to be worthwhile. I mention these here more briefly than in Ref. [52, and also make a couple of new suggestions. Those previously suggested which still seem to be worthwhile are: 1 . Use micro-Hall probes near the sample surface to measure the time and spatial dependence of magnetic fields near the surface. One property which would be determined from such measurements is the direction of the magnetic moment in the channels in metamagnetic samples; 2. Perform magnetisation measurements for fields parallel to sample surfaces. One might expect a decrease of magnetisation with time in such fields as opposed to an increase seen in fields perpendicular to surfaces, attributed to gradual alignment of magnetism in channels in the direction of the film thickness [19; 3. Attempt to study the channels by optical microscopy at oblique incidence in order to have a component of electric field parallel to the channel directions, and with various wavelengths to try to find the size of any energy gap in the electron spectrum; 4. Perform electrical measurements in magnetic fields higher than those required to produce a metamagnetic transition to see whether the high conductivity remains in this region; 5. Perform magnetic measurements below the glass transition temperature to see if time dependent effects observed above this temperature 19 in magnetic fields no longer occur at low $T ; 6$. Perform electrical measurements with films under tension between surfaces to see whether this enables one to find conducting channels parallel to film surfaces.

Another suggestion which would help to test our model, which involves resistance mainly due to phase slips in the smaller-resistance mediumly conducting channels in Fig. 1, would be first to determine histograms of resistance for microcontacts on a suitable film at room temperature, and then to look at the temperature dependence of resistance at points with resistance near the high-resistance end of the range for superconducting channels and at the low-resistance end of the range of the mediumly resistant points. If our interpretation, which involves some resistance due to phase slips, is correct, then the lower-resistance mediumly conducting points would become highly conducting at lower temperatures, while points at the higher-resistance end of the low-resistance points would change to medium resistances at temperatures somewhere between 295 $\mathrm{K}$ and $(295 K+\Delta T)$, where $\Delta T$ corresponds to the change of transition temperature due to phase slips in the highest-resistance superconducting channels for whatever film thickness is being studied. Quantitative analysis of such experiments would enable $\Delta T$ and $T_{c}$ to be determined as a function of $n_{T}$, and so would remove our requirement to make guesses at $\Delta T$. It would also be useful to determine the relation between size of channels and their resistance (in two-probe measurements) if the size of the channels can be determined e.g. 
by electron microscopy. Although the experiments suggested in this paragraph would be quite time comsuming, we think they would go a long way towards establishing superconductivity at room temperature, and whether our model for it is realistic. High resolution electron microsopy could possibly detect the nm-size subsystems of conducting channels used in our model.

New theory needed

Two types of theory which would be useful to develop are: (i) An extension of the Ambegoakar-Langer-McCumber-Halperin theory to help determine amounts of phase slips for Bose-gas superconductors; (ii) Calculations of transverse exchange energies and any magnetic anisotropy in our model. The first of these types of theory is likely to be difficult. To calculate the transverse exchange energy using Grigorov's theory 48 and our parameters should be straightforward, but calculations of magnetic anisotropy and sizes of domains do not appear to be simple.

\section{Conclusions}

We have used a model of Bose condensation of bosons in arrays of nanofilaments to interpret some results on room-temperature superconductivity in narrow channels through films of oxidised atactic polypropylene. The model makes use of a theory of Grigorov and coworkers involving nanofilaments composed of strings of an unusual type of polaron produced by interaction with rotatable dipolar groups in an elastomer, and then assuming that the polarons in the strings bind to form bipolarons due to mediation of high-frequency bosons. These bosons are assumed to be plasmons for most of our calculations, although a fair fit to experimental and theoretical constraints may also be obtained assuming the bosons are high-energy phonons with energy $0.38 \mathrm{eV}$. There are several differences from a model previously used 3 , especially (i) a different method of estimating the numbers of nanofilaments in the highest-resistance superconducting channels, and (ii) a smaller slope of the initial linear term in the dispersion of the bosons than previously used. This slope is based on a Bogoliubov form of dispersion for bosons at $T_{c}$ with a slope determined by requiring that the initial change from the condensation temperature for an ideal Bose gas as the boson concentration is increased is about the same as determined by accurate published calculations of the effects of interactions on $T_{B}$ of a Bose gas. There are twelve parameters in our model. For one parameter related to changes in $T_{c}$ due to phase slips we can only determine a lower limit, and we choose a value slightly higher than this limit. The other parameters can then be determined approximately by use of twelve relations between parameters of varying degrees of estimated accuracy, four of these involving experimental input and eight based on theoretical relations between parameters. Some suggestions for further experiments are made, and also for theory which it would be worthwhile to develop. 


\section{Acknowledgments}

I wish to thank L.N. Grigorov for discussions in person and by e-mail at various times up to early 2007, and A.N. Ionov for correspondence, and for some comments on a draft of the Introduction.

\section{References}

[1] L.N. Grigorov and S.G. Smirnova, Deposited Article No. 2381, All-Union Institute for Scientific and Technological Information, 23 March 1988, V p 88

[2] N.S. Enikolopyan, L.N. Grigorov and S.G. Smirnova, Pis'ma Zh. Eksp. Teor. Fiz. 49, 326 (1989) [JETP Lett. 49, 371 (1989)]

[3] D.M. Eagles, Phil. Mag. 85, 1931 (2005)

[4] V.M. Arkhangorodskii, A.N. Ionov, V.M. Tuchkevich and I.S. Shlimak, Pis'ma Zh. Eksp. Teor. Fiz. 51, 56 (1990) [JETP Lett. 51, 67 (1990)]

[5] O.V. Demicheva, D.N. Rogachev, S.G. Smirnova, E.I. Shklyarova, M.Yu. Yablokov, V.M. Andreev and L.N. Grigorov, Pis'ma Zh. Eksp. Teor. Fiz. 51, 228 (1990) [JETP Lett. 51, 258 (1990)]

[6] L.N. Grigorov, O.V. Demicheva and S.G. Smirnova, Sverkhprovodimost' (KIAE) 4, 399 (1991) [Superconductivity, Phys. Chem. Tech. 4, 345 (1991)]

[7] G.M. Zhao and Y.S. Wang, condmat/0111268

[8] G.M. Zhao, condmat/0307770

[9] G.M. Zhao, in Trends in Nanotube Research, ed. Delores A. Martin (Nova Science, New York, 2006), pp 39-75 (2006)

[10] G.M. Zhao and. P. Beeli, Phys. Rev. B 77, 245433 (2008)

[11] V.I. Tsebro, O.E. Omel'yanovskii and A.P. Moravskii, Pis'ma Zh. Eksp. Teor. Fiz. 70, 457 (1999) [JETP Lett. 70, 462 (1999)]

[12] Y. Kopelevich, P. Esquinazi, J.H.S. Torre and S. Moehlecke, J. Low Temp. Phys. 119, 691 (2000)

[13] Y. Kopelevich and P. Esquinazi, J. Low Temp. Phys. 146, 629 (2007)

[14] D. Djurek, Z. Medunić, A. Tonejc and M. Paljević, Physica C 351, 78 (2001)

[15] D. Djurek, condmat/0811.4352

[16] D. Djurek, J. Supercond. Nov. Mag. 24, 199 (2011) 
[17] S.G. Smirnova, O.V. Demicheva and L.N. Grigorov, Pis'ma Zh. Eksp. Teor. Fiz. 48, 212 (1988) [JETP Lett. 48, 231 (1988)]

[18] L.N. Grigorov, D.N. Rogachev and A.V. Kraev, Vysokomol. Soedin. B 35, 1921 (1993) [Polymer Science 35, 1625 (1993)].

[19] L.N. Grigorov, T.V. Dorofeeva, A.V. Kraev, D.N, Rogachev, O.V. Demicheva and E.I. Shklyarova, Vysokomol. Soedin. A 38, 2011 (1996) [Polymer Science A 38, 1328 (1996)]

[20] D.N. Rogachev and L.N. Grigorov, J. Supercond. 13, 947 (2000)

[21] D.M. Eagles, J. Supercond. 15, 243 (2002)

[22] A.N. Ionov and V.M. Tuchkevich, Pis'ma Zh. Tekh. Fiz. 16 (15-16), 90 (1990) [Sov. Tech. Phys. Lett. 16, 638, (1990)]

[23] A.N. Ionov, A.N. Lachinov, M.M. Rivkin and V.M. Tuchkevich, Solid State Commun. 82, 609 (1992)

[24] A.N. Ionov and V.A. Zakrevskii, Pis'ma Zh. Tekn. Fiz. 26 (20), 34 (2000) [Tech. Phys. Lett. 26, 910 (2000)]

[25] A.N. Ionov, R. Rentzsch and M.N. Nikoleeva, Phys. Stat. Sol. (c) 5, 730 (2008)

[26] R. Rentzsch and A.N. Ionov, Phys. Stat. Sol. (c) 5, 735 (2008)

[27] I. Shlimak and V. Martchenkov, Solid State Commun. 107, 443 (1998).

[28] M. Baran, V.A. Beloshenko, V.P. D’yakonov, É.E. Zukov, A. Nabialek and R. Shimchak, Fiz. Tverd. Tela 37, 3438 (1995) [Sov. Phys. Solid State 37, 1889 (1995)]

[29] P.M. Grant, Physics Today, May 1998, p17

[30] L.N. Grigorov and K.P. Shambrook, US Patent No. 5777292 (1998)

[31] L.N. Grigorov and R.V. Talroze, US Patent No. 6563132 (2003)

[32] L.N. Grigorov and A. Krayev, US Patent No. 7014795 (2006)

[33] A.V. Krayev, T.V. Dorofeeva, E.I. Shklyarova and L.N. Grigorov, 9th. CIMTEC - World Forum on New Materials, Florence, Italy, 14-19 June 1998; Advances in Science and Engineering Technology, Vol. 23: Science and Engineering of HTC Superconductivity, edited by P. Vincennzini (Faenza Techna., Srl., 1999), pp. 459-466.

[34] A.N. Ionov, A.N. Lachinov and R. Rentzsch, Pis'ma Zh. Tekh. Phys. 28 (14), 69 (2002) [Tech. Phys. Lett. 26, 608 (2002)] 
[35] A.N. Ionov, V.A. Zakrevskiĭ, V.M. Svetlichny and R. Rentzsch, 10th. Int. Symp. "Nanostructures: Physics and Technology", St. Petersburg, Russia, June 17-21, 2002 [SPIE Proceedings 5023, 475 (2003)]

[36] A.N. Ionov, V.A. Zakrevskii and I.M. Lazebnik, Pis'ma Zh. Tekh. Phys. 25 (17), 36 (1999) [Tech. Phys. Lett. 25, 691 (1999)]

[37] A.N. Ionov and R. Rentzsch, Annalen der Physik 18, 963 (2009)

[38] A.N. Lachinov, Sensors and Actuators A 39, 1 (1993)

[39] O.A. Scaldin, O.A. Selezneva, Y.A. Lebedev, A.N. Chuvrov, J. Appl. Phys. 77, $3194(1995)$

[40] A.N. Lachinov, Physics-Uspekhi 49, 1223 (2006)

[41] V.A. Zakrevskii, A.N. Ionov and A.N. Lachinov, Pis'ma Zh. Tekh. Fiz. 24 (13), 89 (1998) [Tech. Phys. Lett. 24, 539 (1998)]

[42] A.N. Ionov and R. Rentzsch, in Proc. of SPIE 7521, 752100 (2010) International Conference on Micro- and Nano-Electronics 2009.

[43] A.N. Ionov and R. Rentzsch, to be published.

[44] A.N. Ionov, M.S. Dunaevskii, M.N. Nikolaeva and R. Rentzsch, Annalen der Physik 18, 959 (2009)

[45] A.V. Kraev, S.G. Smirnova and L.N. Grigorov, Vysokomol. Soedin. A 35. 1308 (1993) [Polymer Science 35, 1082 (1993)]

[46] L.N. Grigorov, Makromol. Chem., Macromol. Symp. 37159 (1990)

[47] L.N. Grigorov, V.M. Andreev and S.G. Smirnova, Makromol. Chem., Macromol. Symp. 37177 (1990)

[48] L.N. Grigorov, Pis'ma Zh. Tekh. Fiz. 17 (5), 45 (1991) [Sov. Tech. Phys. Lett. 17368 (1991)]

[49] L.N. Grigorov, Phil. Mag. B 78, 353 (1998).

[50] L.N. Grigorov, 9th. CIMTEC - World Forum on New Materials, Florence, Italy, 14-19 June 1998; Advances in Science and Engineering Technology, Vol. 23: Science and Engineering of HTC Superconductivity, edited by P.Vincennzini (Faenza Techna. Srl., 1999), pp. 675-684

[51] D.M. Eagles, Physica C 225, 222 (1994) ; erratum ibid. 280, 335 (1997)

[52] D.M. Eagles, J. Supercond. 11, 189 (1998)

[53] D.M. Eagles, R.M. Quick and B. Schauer, Phys Rev. B 75, 054305 (2007)

[54] M. Ferrier, A. de Martino, A. Kasumov, S. Guéron, M. Kociak, R. Egger and H. Bouchiat, Solid State Comm. 131, 615 (2004) 
[55] S.K. Adhikari, M. Casas, A. Puente, A. Rigo, M. Fortes, M.A. Solís, M. de Llano, A.A. Valladares and O. Rojo, Phys. Rev. B 62, 8671 (2000)

[56] P. Arnold and G. Moore, Phys. Rev. Lett. 87, 120401 (2001)

[57] V.A. Kashurnikov, N.V. Prokof'ev and B.V. Svistunov, Phys. Rev. Lett. 87, $120402(2001)$

[58] S. Pilati, S. Giorgini and N. Prokof'ev, Phys. Rev. Lett. 100, 140405 (2008)

[59] L.G. Aslamozov and A. Larkin, Fiz. Tverd. Tela 10, 1104 (1968) [Sov. Phys. Solid State 10, 875 (1968)]

[60] A.S. Alexandrov and N.F. Mott, Phys. Rev. Lett. 71, 1075 (1993)

[61] A.N. Ionov, V.M. Svetlichinyi and R. Rentzsch, Physica B 359-361, 506 (2005)

[62] J. Lowell and A.C. Rose-Innes, Adv. Phys. 29, 947 (1980)

[63] D.M. Eagles, Physica C 301, 165 (1998)

[64] V.A.Alekseev, Zh. Éksp. Teor. Fiz. 119, 700 (2001) [JETP 92, 60 (2001)]

[65] V.A. Alekseev, Zh. Éksp. Teor. Fiz. 121, 1273 (2002) [JETP 94, 1091 (2002)]

[66] C, Schönenberger, A. Bachtold, C. Strunk, J.-P. Salvetat and L. Forro, Appl. Phys. A 69, 283 (1999)

[67] J.S. Langer and V. Ambegoakar, Phys. Rev. 164, 498 (1967)

[68] D.E. McCumber and B.I. Halperin, Phys. Rev. B 1, 1054 (1970)

[69] A. Polkovnikov, E. Altman, E. Demler, B.I. Halperin and M.D. Lukin, Phys. Rev. A 71, 063613 (2005)

[70] A. Polkovnikov, E. Altman, E. Demler, B.I. Halperin and M.D. Lukin, J. Supercond. 17, 577 (2005)

[71] I.A. Shelykh, N.T. Bagraev, V.K. Ivanov and L.E. Klyachkin, J. Supercond. 16, 355 (2003)

[72] S.G. Smirnova, E.I. Shklyarova and L.N. Grigorov, Vysokomol. Soedin B 31, 667 (1989)

[73] A. Alvermann, H, Fehske and S.A. Trugman, Phys. Rev. B 78, 165106 (2008)

[74] J.P. Hague, P.E. Kornilovitch, A.S. Alexandrov and J.H. Samson, Physica C 460-462, 1115 (2007)

[75] J.P. Hague and P.E. Kornilovitch, Phys. Rev. B 80, 054301 (2009) 
[76] M. Hohenadler and W. von der Linden, Phys. Rev. B 71, 184309 (2005)

[77] P. Pieri, L. Pisani and G.C. Strinati, Phys. Rev. B 72, 012506 (2005)

[78] C. Chin, Phys. Rev. A 72, 041601 (2005)

[79] L.M. Jensen, H.M. Nilsen and G. Watanabe, Phys. Rev. A 74, 043608 (2006)

[80] D.M. Eagles, Phys. Rev. 186, 456 (1969)

[81] O.V. Demicheva, D.N. Rogachev, V.M. Andreev, E.I. Shklyarova, S.G. Smirnova and L.N. Grigorov, Vysokomol. Soedin. 32 (1), 4 (1990)

[82] H. Heiselberg, Phys. Rev. A 73, 013607 (2006)

[83] D.S. Petrov, C. Salomon and G.V. Shlyapnikov, Phys. Rev. Lett. 93, 090404 (2004)

[84] P. Pieri, L. Pisani and G.C. Strinati, Phys. Rev. B 70, 094508 (2004)

[85] W.H. Press, B.P. Flannery, S.A. Teukolsky and W.T. Vetterling, Numerical Recipes, Cambridge University Press (1986)

[86] J.A. Nelder and R. Mead, Computer Journal 7, 308 (1965)

[87] F.V. Kusmartsev, Contemp. Phys. 45, 237 (2004)

[88] A.S. Alexandrov and V.V. Kabanov, Pis'ma Zh.Eksp.Teor.Fiz. 72, 825 (2000) [JETP Lett. 72, 569 (2000)]

[89] J.P. Hague, P.E. Kornilovitch, J.H. Samson and A.S. Alexandrov, Phys. Rev. Lett. 98, 037002 (2007)

[90] E. Lieb and D. Mattis, Phys. Rev. 125, 164 (1962)

[91] J.S. Meyer and K.A. Matveev, J. Phys.: Cond. Mat. 21, 023203 (2009)

[92] M.P. McDonald and I.M. Ward, Polymer 2, 241 (1961) 